PROCEEDINGS OF THE

AMERICAN MATHEMATICAL SOCIETY

Volume 129, Number 6, Pages 1665-1668

S 0002-9939(00)06003-2

Article electronically published on October 25, 2000

\title{
LINEAR MAPS PRESERVING IDEALS OF $C^{*}$-ALGEBRAS
}

\author{
SANG OG KIM
}

(Communicated by Joseph A. Ball)

\begin{abstract}
We show that unital self-adjoint linear bijections of matrix algebras, type $I I_{1}$ factors and abelian $C^{*}$-algebras preserving maximal left ideals are isomorphisms and we show that a unital continuous linear map of a $C^{*}$ algebra $A$ that maps the minimal left ideal $A p$ into itself is the identity map.
\end{abstract}

\section{INTRODUCTION}

Linear preserver problems represent one of the most active research topics in matrix theory. In the last decade considerable attention has also been paid to similar questions in infinite dimension, that is, to linear preserver problems on operator algebras. In both cases, the problem is to characterize those linear maps on the algebra which leave invariant a given subset, or relation, or function. One of the most important such questions concerns the rank. This is because in many cases preserver problems can be reduced to the problem of rank preservers. Also, such problems on ideals were considered. In [3, Theorem 4] they showed that any linear bijection $T: B(H) \rightarrow B(H)$ preserving left ideals in both directions is of the form $T(X)=A X B$ for some invertible elements $A, B \in B(H)$. In the proof they used only the preservation of two extreme kinds of left ideals, namely, that of the minimal ones and that of maximal ones. Motivated by the above theorem, in this paper we consider linear maps of $C^{*}$-algebras that preserve the maximal left ideals and minimal left ideals in one direction or both directions.

\section{Maximal Left ideAl PRESERVERS}

Let us recall some necessary definitions. Let $A$ and $B$ be $C^{*}$-algebras. A linear map $T: A \rightarrow B$ is called self-adjoint if $T\left(a^{*}\right)=T(a)^{*}$ for all $a$ in $A$. If $T: A \rightarrow B$ a linear self-adjoint map such that $T\left(a^{2}\right)=T(a)^{2}$ for every self-adjoint $a$ in $A$, then it is called a $C^{*}$-homomorphism. A $C^{*}$-algebra $A$ is called finite if $a^{*} a=1$, then $a a^{*}=1$. $C^{*}$-algebras with finite traces and those with dense subset of invertible elements are examples of finite $C^{*}$-algebras. Not every $C^{*}$-algebra is finite. For example if $H$ is an infinite dimensional Hilbert space, $B(H)$ and the $C^{*}$-algebra generated by two nonunitary isometries on $H$ are not finite. Note that in a finite $C^{*}$-algebra, every left invertible element is right invertible. Preserving maximal left ideals is connected with the problem of left invertibility preservers. Because of the great interest in linear maps preserving invertibility in one direction or in both

Received by the editors August 31, 1999.

2000 Mathematics Subject Classification. Primary 47B49.

Key words and phrases. $C^{*}$-algebra, derivation, minimal ideal.

(C)2000 American Mathematical Society 
directions we consider linear maps that preserve the maximal left ideals. Unless otherwise stated, we assume that every $C^{*}$-algebra has the identity element.

Lemma 2.1. Let $A$ be a finite $C^{*}$-algebra. If $T: A \rightarrow A$ is a unital self-adjoint linear bijection that preserves the maximal left ideals in both directions, then it is a $C^{*}$-homomorphism.

Proof. Since every proper left ideal is contained in a maximal left ideal, an element fails to have a left inverse if and only if the element is included in a maximal left ideal. Hence $T$ maps the left invertible elements to left invertible elements. Since $A$ is finite every one-sided invertible element of $A$ is invertible. Hence $T$ maps invertible elements of $A$ to such elements of $A$. Then by [1, Theorem 6], $T$ is a $C^{*}$-homomorphism.

Note that a finite simple von Neumann algebra is either a matrix algebra over $\mathbf{C}$ or type $I I_{1}$ factor. For them we have the following lemma.

Lemma 2.2. Let $A$ be a finite simple von Neumann algebra. If $T: A \rightarrow A$ is a unital self-adjoint linear bijection that preserves the maximal left ideals in both directions, then it is either $a^{*}$-isomorphism or a ${ }^{*}$-antiisomorphism.

Proof. Since $T$ is a $C^{*}$-homomorphism by Lemma 2.1, there exist two orthogonal projections $E$ and $F$ in the center of $A$ such that $T_{1}: a \rightarrow T(a) E$ is a ${ }_{-}$ homomorphism (respectively, $T_{2}: a \rightarrow T(a) F$ is an ${ }^{*}$-antihomomorphism), $E+F=$ 1 and $T=T_{1}+T_{2}$ as linear maps by [5, Theorem 3.3]. Since $A$ is simple, 0 and 1 are the only central projections. Hence $T$ is either a ${ }^{*}$-homomorphism or a ${ }_{\text {- }}$ antihomomorphism. This completes the proof.

Theorem 2.3. Let $A$ be finite simple von Neumann algebra acting on a separable Hilbert space $H$, i.e., let $A$ be $M_{n}(C)$ or factor of type $I I_{1}$. If $T: A \rightarrow A$ is a unital self-adjoint linear bijection that preserves the maximal left ideals in both directions, then there exists a unitary $u: H \rightarrow H$ such that

$$
T(a)=u a u^{-1} \text { for } a \in A .
$$

Proof. $T$ is either a ${ }^{*}$-isomorphism or a ${ }^{*}$-antiisomorphism by Lemma 2.2. Assume that $T$ is a ${ }^{*}$-antiisomorphism and let $I$ be a maximal left ideal of $A$. Taking any $b$ in $I$ and $a$ in $A$, since $T(b) T(a)=T(a b) \in T(I), T(I)$ is a right ideal. This implies that $T(I)$ is a two-sided ideal for each maximal left ideal $I$ of $A$. Since $A$ is simple, $\{0\}$ and $A$ are the only closed two-sided ideals. So, this cannot occur. Hence $T$ is a ${ }^{*}$-isomorphism. It is well known that automorphisms of $A$ have such forms.

Now let us consider abelian $C^{*}$-algebras and linear bijections that preserve the maximal ideals. For a linear transformation that maps maximal left ideal into itself, in [2, Theorem 1] they showed that it is a module map. More precisely, they showed the following lemma.

Lemma 2.4 ([2]). Let $A$ be a semi-simple Banach algebra over $\mathbf{C}$ and let $T$ be a linear transformation $A \rightarrow A$ such that $T(I) \subset I$ for each maximal modular left ideal $I$ of $A$. Then $T(a b)=T(a) b$ for all $a, b \in A$; in particular, $T(a)=T(1) a$ for $a \in A$.

Theorem 2.5. Let $A$ be an abelian $C^{*}$-algebra and $T: A \rightarrow A$ a linear bijection that maps the maximal ideals to maximal ideals. Then there is an invertible element 
$v$ in A such that

$$
T(a b)=v T(a) T(b) \text { for all } a, b \in A .
$$

Proof. Let $I \subset A$ be a maximal ideal and denote $J$ the maximal ideal $T(I)$. Let $a \in A$ be an invertible element of $A$ and consider $\psi(a): A / J \rightarrow A / J$ given by $T(b)+J \rightarrow T(a b)+J$. Clearly it is a linear bijection. Since $A / J$ is simple we have by Lemma 2.4

$$
\begin{aligned}
\psi(a)(T(b)+J) & =\psi(a)((1+J))(T(b)+J) \\
& =\psi(a)\left(T\left(T^{-1}(1)\right)+J\right)(T(b)+J) \\
& =T\left(a T^{-1}(1)\right) T(b)+J
\end{aligned}
$$

Hence

$$
T(a b)-T\left(a T^{-1}(1)\right) T(b) \in \bigcap J
$$

This shows that $T(a b)=T\left(a T^{-1}(1)\right) T(b)$ for every invertible element $a \in A$ and every element $b \in A$. Since every element of a $C^{*}$-algebra is a linear combination of invertible ones, it holds for every $a, b \in A$. Noting that $T(a)=T\left(a T^{-1}(1)\right) T(1)$, we have

$$
T(a b)=T\left(T^{-1}(1) a\right) T(b)=T\left(T^{-1}(1) T^{-1}(1)\right) T(a) T(b) .
$$

Since

$$
1=T\left(T^{-1}(1)\right)=T\left(T^{-1}(1) T^{-1}(1)\right) T(1),
$$

$T(1)$ is invertible and $T(1)^{-1}=T\left(T^{-1}(1) T^{-1}(1)\right)$. Taking $v=T(1)^{-1}$, the proof is completed.

Remark. In the proof of the above Theorem, Lemma 2.4 is in fact not necessary because $A / J$ is isomorphic to C. However we list it in connection with Corollary 3.2 .

\section{Minimal Left ideal preservers}

Let $L(A)$ be the set of all bounded linear maps $T: A \rightarrow A$ for a $C^{*}$-algebra $A$. An operator $D \in L(A)$ is called a derivation if

$$
D(x y)=x D(y)+D(x) y
$$

for any $x, y \in A$. For $b \in A$ the left multiplication operator $l_{b}: A \rightarrow A$ acts by the rule $l_{b}(a)=a b$. The idea for the proof of [4, Theorem 1] is used to prove the following theorem. They used it to show that every local derivation of a $C^{*}$-algebra is a derivation.

Theorem 3.1. Let $A$ be a $C^{*}$-algebra such that the linear span of its projections is dense in $A$ and $T \in L(A)$ be such that $T(p A q) \subset p A+A q$ for every projection $p, q \in A$. Then $T(a)=D(a)+a T(1)$ for each $a \in A$, where $D$ is a derivation.

Proof. We may suppose that $T(1)=0$ by considering $T-l_{T(1)}$. Writing $p^{\perp}=1-p$ we have

$$
p^{\perp} T(p A q) q^{\perp}=0
$$

for any projections $p, q \in A$. Now use the obvious identities

$$
p^{\perp} S(p x)-p S\left(p^{\perp} x\right)=S(p x)-p S(x),
$$




$$
V(x q) q^{\perp}-V\left(x q^{\perp}\right) q=V(x q)-V(x) q,
$$

where $S$ and $V$ are arbitrary linear operators in $A$. For $S(x)=T(x q) q^{\perp},(1)$ and (2) imply

$$
T(p x q) q^{\perp}-p T(x q) q^{\perp}=0 .
$$

So for $V(x)=T(p x)-p T(x)$ we have $V\left(x q^{\perp}\right) q=0, V(x q) q^{\perp}=0$ and by (3), $V(x q)-V(x) q=0$. Therefore

$$
T(p x q)-p T(x q)-T(p x) q+p T(x) q=0 .
$$

For $x=1$ we get $T(p q)=p T(q)+T(p) q$ and hence $T(x y)=x T(y)+T(x) y$ for every $x, y \in A$. This completes the proof.

As stated in Lemma 2.4, they showed the form of any linear map of $A$ that maps any maximal left ideal into itself. Now we have an expression for linear maps that preserve the minimal left ideals. Note that any minimal left ideal of $A$ is of the form $A p$ for some projection $p \in A$.

Corollary 3.2. Let $A$ be a $C^{*}$-algebra such that the linear span of its projections is dense in $A$ and $T \in L(A)$ be unital and that $T(A p) \subset A p$ for every projection $p \in A$. Then $T(a)=a$ for each $a \in A$.

Proof. Let $p, q \in A$ be projections. Since $T(q A p) \subset T(A p) \subset A p \subset q A+A p, T(a)=$ $D(a)+a$ for some derivation $D$. We want to show that $D=0$. Let $a \in A$ and $p \in A$ any projection of $A$. Since $T(a p)=D(a p)+a p=D(a) p+a D(p)+a p \in A p$, we have $D(p) \in A p$ for every projection $p \in A$. Since $D(1-p)=-D(p), D(p)=a p=b(1-p)$ for some $a, b \in A$. This shows that $D(p)=0$ for every projection $p \in A$, completing the proof.

\section{REFERENCES}

1. M-D. Choi et. al., On positive linear maps preserving invertibility, J. Funct. Anal. 59 (1984), 462-469. MR 86a:46071

2. B. E. Johnson, Centralizers and operators reduced by maximal ideals, J. London Math. Soc. 43 (1968), 231-233. MR 36:6937

3. L. Molnár, Some linear preserver problems on $B(H)$ concerning rank and corank, Linear Algebra Appl. 286 (1999), 311-321. MR 2000b:47089

4. V. S. Shul'man, Operators preserving ideals in $C^{*}$-algebras, Studia Math. 109(1) (1994), 67-72. MR 95b:46097

5. E. Størmer, On the Jordan structure of $C^{*}$-algebras, Trans. Amer. Math. Soc. 120 (1965), 438-447. MR 32:2930

Department of Mathematics, Hallym University, Chuncheon 200-702, Korea

E-mail address: sokim@sun.hallym.ac.kr 